\title{
ON SEQUENCES NOT CONTAINING A LARGE SUM-FREE SUBSEQUENCE
}

\author{
S. L. G. CHOI
}

\begin{abstract}
A subsequence of a sequence of integers is said to be sum-free if no integer of the subsequence is the sum of distinct integers of this same subsequence. In this paper we shall prove, provided $n$ is sufficiently large, that there exists a sequence of $n$ integers whose largest sum-free subsequence has at most $c n(\log \log n)^{-1 / 2}$ integers, where $c$ is an absolute constant.
\end{abstract}

Given a sequence of $n$ distinct integers

$$
a_{1}<\cdots<a_{n}
$$

we ask for the largest subsequence which is sum-free, i.e., a subsequence no integer of which is the sum of distinct integers of this same subsequence. In [1], Erdös announced the result that there exists a sequence (1) such that the largest sum-free subsequence has at most $o(n)$ integers. Erdös did not give a proof of his result there or subsequently, only mentioning that the construction is complicated; apparently, he has also completely forgotten his proof. In this note, we establish a result which implies that of Erdös by a fairly simple construction; in fact, we shall prove the following

THEOREM. Provided $n \geqq n_{0}$, there exists a sequence of $n$ distinct integers whose largest sum-free subsequence has at most cn $(\log \log n)^{-1 / 2}$ integers, where $c$ is an absolute constant.

For our construction, we shall require a modified form (see lemma below) of a theorem of Varnavides [2] which asserts (essentially) that any sequence of $\delta n$ positive integers not exceeding $n$ contains at least $C_{\delta} x^{2}$ triples in arithmetic progression, where $C_{\delta}$ depends only on $\delta$. Varnavides' result is based on the following theorem of Roth.

THEOREM A. Let $b_{1}, \cdots, b_{n}$ be distinct positive integers not exceeding $x$ so that no three distinct terms are in arithmetic progression. Then there exists an absolute constant $C$ so that $n<C x(\log \log x)^{-1}$.

We now state the necessary modified version of Varnavides' theorem.

Received by the editors March 20, 1973.

AMS (MOS) subject classifications (1970). Primary 10L10.

Key words and phrases. Sum-free, subsequence, arithmetic progressions.

(c) American Mathematical Society 1973 
Lemma. Let $C$ be the constant appearing in Theorem $\mathrm{A}$ and suppose $x \geqq x_{0}(C)$. Let $a_{1}, \cdots, a_{n}$ be any set of distinct positive integers not exceeding $x$, where $n \geqq 3 C x(\log \log x)^{-1}$. Then the number of solutions of

$$
a_{i}+a_{j}=2 a_{h} \quad(i \neq j)
$$

is at least $x^{2} \exp \left\{-c_{1}(\log x)^{1 / 2}\right\}$, where $c_{1}$ is an absolute constant.

The following corollary is an immediate consequence.

COROLlaRY. Let the hypotheses in the lemma be satisfied. Then the number of solutions of (2) is at least $x^{3 / 2}$, provided only $x \geqq x_{0}$.

Before proving the lemma, we remark that only the corollary is used in the proof of our theorem and that the assertion in the lemma is not the strongest that can be proved under the hypotheses. It should also be mentioned that we follow very closely Varnavides' method in proving our lemma.

Proof of lemma. We put

$$
k=\left[\exp \left\{(\log x)^{1 / 2}\right\}\right]+1
$$

so that in particular

$$
\log \log k>\frac{1}{2} \log \log x .
$$

We consider all arithmetic progressions of $k$ terms $1 \leqq u, u+d, \cdots, u+$ $(k-1) d \leqq x$ and call good progressions those which contain at least $C k(\log \log k)^{-1} a$ 's. We let

$$
d<3 C x(\log \log x)^{-1} k^{-2} .
$$

We shall show that the number $G_{d}(x)$ of good progressions, with $d$ fixed and $u$ variable, satisfies

$$
G_{d}(x)>\frac{1}{2} C x(\log \log x)^{-1} .
$$

In fact, every $a_{i}$ which is not less than $k d$ or greater than $x-k d$ is contained in exactly $k$ progressions. The number of these $a_{i}$ is at least

$$
3 C x(\log \log x)^{-1}-2 k d \geqq \frac{5}{2} C x(\log \log x)^{-1},
$$

in view of (4).

Denote by $f(u, d)$ the number of $a$ 's in the progression $u, u+d, \cdots, u+$ $(k-1) d$; then

$$
\sum_{u} f(u, d)>\frac{5}{2} C x k(\log \log x)^{-1},
$$

where the summation is extended over $u \geqq 1, u+(k-1) d \leqq x$. The number 
of progressions with $u \geqq 1, u+(k-1) d \leqq x$, is clearly less than $x$ and thus we have

$$
\sum_{u} f(u, d)<C x k(\log \log k)^{-1}+G_{d}(x) k .
$$

Combining (6) and (7), and making use of (3)', we obtain (5).

Now, if $G(x)$ denotes the total number of good progressions for all integers $d$ satisfying (4), then we have

$$
G(x)>(C x /(k \log \log x))^{2} .
$$

Each good progression yields a solution of (2) in view of Theorem A. Further, it is easy to see that the same solution $\left(a_{i}, a_{h}, a_{j}\right)$ of (2) can occur in at most $k^{2}$ progressions. So the number of solutions is, on using (8) and (3), at least $k^{-2} G(x)>x^{2} \exp \left\{-c_{1}(\log x)^{1 / 2}\right\}$, where $c_{1}$ is an absolute constant. This completes the proof of the lemma.

We are now in a position to prove our theorem.

Let $n \geqq n_{0}$ be given. We put

$$
t=\left[n(\log \log n)^{-1 / 2}\right]
$$

and determine $s$ so that

$$
t(s+1) \leqq n<t(s+2) .
$$

We define a set $\mathscr{A}$ of $n$ integers as follows: ${ }^{1}$ For each $i=0,1, \cdots, s$, let

$$
\mathscr{A}_{i}=2^{i}[t, 2 t) \text {. }
$$

Let $\mathscr{A}_{s+1}$ consist of any $n-t(s+1)$ integers distinct from those in $\mathscr{A}_{0}, \cdots$, $\mathscr{A}_{s}$. Finally, put

$$
\mathscr{A}=\mathscr{A}_{0} \cup \mathscr{A}_{1} \cup \cdots \cup \mathscr{A}_{s} \cup \mathscr{A}_{s+1} \text {. }
$$

Suppose $\mathscr{B}$ is a sum-free set from $\mathscr{A}$. We shall establish the theorem by showing that

$$
|\mathscr{B}| \ll n(\log \log n)^{-1 / 2},
$$

where the implied constant is absolute. For each $i=0, \cdots, s$, we denote by $\mathscr{B}_{i}$ the intersection of $\mathscr{A}_{i}$ and $\mathscr{B}$, i.e. $\mathscr{A}_{i} \cap \mathscr{B}$. Let $\mathscr{B}_{i_{1}}, \mathscr{B}_{i_{2}}, \cdots, \mathscr{B}_{i_{u}}$ be those $\mathscr{B}_{i}$ each with at least $t^{1 / 2}$ integers, say. Consider the union of

$$
2^{s-i_{1}} \mathscr{B}_{i_{1}}, \cdots, 2^{s-i_{u}} \mathscr{B}_{i_{u}} ;
$$

all these integers lie in $\mathscr{A}_{s}$ since, by (11), any integer in $\mathscr{A}_{i}$ is divisible

\footnotetext{
${ }^{1}$ For real numbers $\alpha>\beta$ and integer $y$, we use $y[\alpha, \beta)$ to denote the set of integers $y m$, where $m$ runs through all integers in $[\alpha, \beta)$.
} 
by $2^{i}$. We shall show that, whenever $1 \leqq j<l \leqq u$, we have

$$
\left|2^{s-i j} \mathscr{B}_{i_{j}} \cap 2^{s-i l} \mathscr{B}_{i_{l}}\right|<3 C t(\log \log t)^{-1} \text {. }
$$

We note that (13) immediately implies (12), since (13) implies

$$
\left|\mathscr{B}_{i_{1}}\right|+\cdots+\left|\mathscr{B}_{i_{u}}\right| \leqq 3 u^{2} C t(\log \log t)^{-1}+t \leqq 3 s^{2} C t(\log \log t)^{-1}+t \text {, }
$$

and consequently,

$$
\begin{aligned}
|\mathscr{B}| & =\left|\mathscr{B}_{0}\right|+\cdots+\left|\mathscr{B}_{s+1}\right| \\
& \leqq t^{1 / 2}(s+1)+\left|\mathscr{B}_{s+1}\right|+\left|\mathscr{B}_{i_{1}}\right|+\cdots+\left|\mathscr{B}_{i_{u}}\right| \\
& \leqq t^{1 / 2}(s+1)+t+3 s^{2} C t(\log \log t)^{-1}+t,
\end{aligned}
$$

which, in view of (9) and (10), is

$$
\begin{aligned}
& \leqq 3 t+(2 \log \log n)(3 C t / \log \log n) \\
& \ll t<n(\log \log n)^{-1 / 2} .
\end{aligned}
$$

To complete the proof of the theorem, it remains to establish (13). Suppose, on the contrary, that (13) is not true for some $j, l$ satisfying $1 \leqq j<l \leqq u$. Then there exist integers $a_{1}, \cdots, a_{v}, v \geqq 3 C t(\log \log t)^{-1}$, in $\mathscr{B}_{i j}$, so that

$$
2^{i^{i}-i_{j}} a_{1}, \cdots, 2^{i l^{-i j}} a_{v}
$$

appear in $\mathscr{B}_{i_{l}}$. By the corollary to the lemma, there exists some $a^{*} \in \mathscr{B}_{i_{1}}$ such that $a_{v_{1}}+a_{w_{2}}=2 a^{*}$ has at least $t^{1 / 2}$ solutions in $a_{1}, \cdots, a_{v}$. Since $2^{i_{l}-i_{j}-1} \leqq 2^{8} \leqq t^{1 / 2}$, by taking $2^{i_{l}-i_{j}-1}$ such pairs we obtain a sum $2^{i_{l-i} a^{*}}$, which is an integer in $\mathscr{B}_{i_{i}}$. This violates the assumption that $\mathscr{B}$ is sum-free. This contradiction completes the proof of the theorem.

To conclude, we note that any improvement on the upper bound $C n(\log \log n)^{-1}$ occurring in Theorem A will, of course, result in a corresponding improvement of the bound in our theorem.

\section{REFERENCES}

1. P. Erdös, Extremal problems in number theory, Proc. Sympos. Pure Math., vol. 8, Amer. Math. Soc., Providence, R.I., 1965, pp. 181-189. MR 30 \#4740.

2. P. Varnavides, On certain sets of positive density, J. London Math. Soc. 34 (1959), 358-360. MR 21 \#5595.

Department of Mathematics, University of British Columbia, Vancouver, British Columbia, Canada 